http://dx.doi.org/10.18778/2196-8403.2007.14

\author{
LESŁAW TOBIASZ
}

\title{
Wortschatzarbeit in alternativen Lehrmethoden eine kritische Betrachtung
}

W artykule przeprowadzona została krytyczna analiza pracy ze słownictwem w wybranych alternatywnych metodach nauczania języków obcych (sugestopedia, Silent Way, Community Language Learning, Total Physical Response). W metodach tych daje się zauważyć przypisywanie wyjątkowego znaczenia czynnikowi emocjonalnemu jako katalizatorowi przyswajania nowych wiadomości. Powoduje to zawężone postrzeganie złożoności procesu uczenia się języka i zmniejsza efektywność nauczania alternatywnego.

Der Artikel setzt sich mit der Wortschatzarbeit in ausgewählten alternativen Lehrverfahren kritisch auseinander (Suggestopädie, Silent Way, Community Language Learning, Total Physical Response). In diesen Methoden wird die Bedeutung emotiver Faktoren als Katalysatoren des Erwerbens neuen Wissens besonders stark hervorgehoben, was eine eingeengte Sichtweise auf die Komplexität der fremdsprachlichen Lernprozesse und damit eine verminderte Effektivität des alternativen Sprachvermittelns bedeutet.

The article critically analyses learning of vocabulary in selected alternative methods of foreign language teaching (Suggestopaedia, Silent Way, Community Language Learning, Total Physical Response). In these methods special emphasis is placed on the significance of emotions seen as catalysts of knowledge acquisition. This results in a restricted perception of the complexity of foreign language learning and limits the efficiency of alternative language instruction. 


\section{Einleitung}

Dem Wortschatz kommt in allen Spracherwerbstypen eine sehr wichtige Rolle zu. Beim L1-Erwerb erfassen die Kinder dank der Versprachlichung mentaler Konzepte allmählich die Welt und leiten die syntaktischen Strukturen von semantischen ab. Beim natürlichen L2-Erwerb ermöglichen die Wörter die kommunikative Interaktion bei fehlerhafter Aussprache und Syntax. Auch im Fremdsprachenunterricht ist die Bedeutung der zielsprachlichen Vokabeln für die Lernfortschritte kaum zu unterschätzen. Dieser Tatsache wird in der letzten Zeit Rechung getragen durch den höheren Stellenwert, den Textarbeit und Wortschatzübungen in Sprachlehrwerken erhalten haben (BOHN / SCHREITER 2000). Gleichwohl treten bei der Wortschatzarbeit immer wieder Probleme auf, die besonders das dauerhafte, interferenzfreie Einprägen und den aktiven Gebrauch neuer Wörter in der Kommunikation betreffen. Es gibt aber auch Mängel in der Beherrschung des rezeptiven Wortschatzes, was besonders im unzureichenden Verstehen neuer, gelesener oder gehörter Texte zum Ausdruck kommt. Solche im Unterrichtsalltag leicht zu beobachtenden Lernschwierigkeiten, die selbstverständlich nicht nur lexikalischer Natur sind, sondern auch in der mangelhaften Beherrschung der fremdsprachlichen Morphosyntax ihren Ursprung haben, waren in den 70er und 80er Jahren des 20. Jahrhunderts (besonders in den USA) ein wichtiger Grund für die Entwicklung alternativer Lehr- und Lernmethoden. Ihr theoretisches Gerüst stützt sich auf die Ergebnisse der Gedächtnisforschung, der menschlichen Kognition und der Psychologie und basiert auf der Beobachtung der Unterrichtspraxis. Im vorliegenden Beitrag werden die wichtigsten alternativen Methoden wie Suggestopädie, The Silent Way, Community Language Learning und Total Physical Response hinsichtlich der Wortschatzarbeit einer kritischen Analyse unterzogen. Untersucht werden soll die Frage, ob sich wirklich eine ,moderne" Methode etablieren kann, die sich als Allheilmittel gegen alle bisherigen lexikalischen Probleme im Fremdsprachenunterricht einsetzen ließe oder ob die alternativen Methoden eher eine modische Erscheinung darstellen, die als eine Art Abwechslung im herkömmlichen Unterricht zu betrachten sind.

\section{Suggestopädie}

Die Suggestopädie nimmt unter den alternativen Methoden eine besondere Stellung ein. Sie entstand im Unterschied zu den anderen neuen Lehrkonzepten nicht in den USA, sondern wurde als Lehr- und Lernmethode in den 1960er Jahren von dem bulgarischen Arzt und Psychotherapeuten Georgi Lozanov 
entwickelt. Sie war primär nicht für den Fremdsprachenunterricht gedacht. Lozanov interessierte eigentlich nur die Frage, , ,...] inwieweit die Gedächtnisleistung von Menschen durch die Übermittlung von Informationen erweitert werden kann, die von einem Lerner unbewusst aufgenommen werden“" (BAUR 1991:7). Erst später entstand ein entsprechendes Konzept für den Fremdsprachenunterricht. Weil aber die Suggestopädie als eine universale Lehrmethode angesehen wird, wird sie auch in anderen Fächern, wie z. B. Mathematik oder Geographie, eingesetzt (zu verschiedenen Einsatzmöglichkeiten der Suggestopädie BRÖHM-OFFERMANN 1994:58-121).

Auch im Fremdsprachenunterricht besteht die Aufgabe der Suggestopädie im Schaffen einer Lehr- und Lernatmosphäre, in der sowohl bewusste als auch unbewusste Wahrnehmungskanäle des Lerners aktiviert werden und somit eine Verknüpfung von Kognition und Affekt stattfindet. Dies geschieht zuerst durch den Abbau der psychischen Hemmungen des Lernenden. Er wird in den Zustand einer kindlichen Lernbegeisterung versetzt. Diese Infantilisierung wird jedoch erst dann erreicht, wenn sich der Lerner völlig entspannt und sich dem Lehrenden anvertraut. „Dieses Vertrauen gibt dem Lerner innere Ruhe, steigert seine Aufnahmebereitschaft und seine Bereitschaft zum Spiel, das in der Suggestopädie eine wichtige Funktion hat." (BAUR 1991a:297) Die aus dieser Passivität resultierende Steigerung der Aufnahmebereitschaft ist oft so erheblich, dass schon im Anfangsunterricht mit sehr vielen lexikalischen zielsprachlichen Einheiten operiert wird (200-400 lexikalische Einheiten in der ersten Lektion), was eine frühe Kommunikation in der Fremdsprache ermöglicht (BAUR 1991a:291-293). Die verwendeten Semantisierungs- und Behaltensverfahren schließen Übersetzungen in die Muttersprache, Visualisierungen, Gestik, Mimik, Tafelbilder, assoziative Arbeit mit semantischen Netzwerken, Sprachlernspiele, Körperbewegungen (darunter Ausführung von Handlungen), rhythmisches Lesen und Sprechpausen ein. Der Lehrer sollte „versuchen, so viele sensorische Perzeptionskanäle anzusprechen wie möglich und dabei auch begleitende Gesten und Mimik zu verwenden, die von den Schülern unmittelbar imitiert werden können“ (HOLTWISCH 1994:230). Die Lerner prägen sich die zielsprachlichen Vokabeln oft ein, indem sie den fremdsprachlichen Text mit geschlossenen Augen hören und sich die einzelnen Handlungsabläufe vor ihrem inneren Auge vorstellen. „Für diese Visualisierungstechnik eignen sich vor allem Texte, die handlungsorientierte Abläufe enthalten und sich deshalb gut verbildlichen lassen." (HOLTWISCH 1994:230) Eine sehr wichtige Rolle spielt die Musik (zwei Minuten musikalische Einführung - z. B. eine Sarabande -, dann 25 Minuten Barockmusik), die einerseits die Entspannung der Lerner, andererseits aber eine unbewusste Aufnahme des 
zielsprachlichen Materials ermöglicht. Während nämlich die Barockmusik erklingt, spielt der Lehrer den Dialog vor. Die Lerner konzentrieren sich jedoch nur auf die Musik, wobei sie ihre Augen geschlossen haben. Den abschließenden Teil bilden zwei Minuten eines schnellen Satzes, der die Lerner aus ihrer Trance zurückholt (LEWICKI 1990:51-56).

Testergebnissen zufolge scheint das suggestopädische Lehrverfahren in hohem Maße das Behalten und die Produktion der fremdsprachlichen lexikalischen Strukturen zu fördern. Dies ist nicht zuletzt darauf zurückzuführen, dass dieses innovative Lehrkonzept die Erkenntnisse der Psycho- und Neurolinguistik sowie der Humanistischen Psychologie mitberücksichtigt, was z. B. in der stressfreien Atmosphäre, in der Freisetzung der positiven Emotionen und im mehrkanaligen Lernen zum Ausdruck kommt (SCHIFFLER 1989). Die Suggestopädie ermöglicht zudem eine Persönlichkeitsentfaltung des Lerners, die in der herkömmlichen Unterrichtspraxis kaum vorstellbar ist (RIEDEL 2000). Die erwähnten Testergebnisse sollten dennoch mit einer gewissen Vorsicht bewertet werden. Die Zusammensetzung der suggestopädisch unterrichteten Gruppen ist nämlich durch den Kurs weitgehend vorbestimmt. Außerdem werden die Lerntechniken der Suggestopädie fast ausschließlich in Erwachsenengruppen im Zusammenhang mit Intensivkursen eingesetzt. Im schulischen Fremdsprachenunterricht hat man sie bisher wenig erprobt. Nicht zu übersehen ist auch die Tatsache, dass diese alternative Lehrmethode eine erfolgreiche Anwendung hauptsächlich im Anfangsunterricht findet, später indes ohne reflektiertes Lernen nur schwer praktikabel ist. Einer größeren Verbreitung der Suggestopädie stehen nicht zuletzt überdurchschnittliche Anforderungen an den Lehrer im Wege. Er muss sowohl schauspielerische Begabung als auch psychologische Grundkenntnisse besitzen. Er sollte ein positives autoritäres Verhalten zeigen und zugleich durch nichts in seiner Stimme, Körperhaltung, Gestik die entspannte Atmosphäre zerstören (LEWICKI 1990:57-61, 170-173).

\section{Silent Way}

Silent Way (,Der stille Weg') wurde von CALEB GATtegno (1978) entwickelt und war zunächst für den Mathematikunterricht bestimmt. Durch dieses Lehrverfahren soll der Lerner dazu befähigt werden, seine kognitiven Kräfte voll zu entfalten. Dies ist nach Gattegno erst dann möglich, wenn der Lerner selbständig die zielsprachlichen Regeln entdeckt und das Vertrauen in die Richtigkeit der auszusprechenden Wörter gewinnt. Damit dieses autonome und verantwortungsvolle Lernen stattfindet, muss sich der Lehrer dem Lerner unterordnen. „Er darf die Entfaltung des Kriterions nicht durch seine eigenen Äußerun- 
gen behindern. Er bringt in das Unterrichtsgeschehen seine Kenntnis der Sprache ein und lenkt stumm das Unterrichtsgeschehen, die Aktivitäten.“ (SCHWERDTFEGER 1983:7) Seine Aufgabe besteht vor allem darin, eine Lernsituation zu schaffen, in der die Lerner ihre kognitiven Lernstrategien optimal anwenden. Weil der Lehrer möglichst keine fremdsprachlichen Äußerungen produzieren soll, unterstützt er korrektes sprachliches Schülerverhalten durch Gestik bzw. Mimik. Eine wichtige Rolle spielt in dem Lehrkonzept die Übung der Aussprache zielsprachlicher Laute und die Ausführung von Handlungen mit farbigen Cuisenaire-Stäbchen (Bezeichnung nach dem belgischen Mathematiker, der sie entwickelt hat). Den einzelnen Lauten werden dabei verschiedene Farben zugeordnet, und die Handlungen werden versprachlicht. Weil jedoch Gattegno für die Anfangsphase des Unterrichts keine besonderen Formen der Wortschatzarbeit vorsieht, helfen dem Lerner bei der Produktion der Äußerungen in der Fremdsprache fertige große Tafeln, auf denen die zielsprachlichen Wörter in den entsprechenden Farbcodes dargestellt sind. Diese Tafeln werden nach und nach an die Wände des Unterrichtsraums gehängt. So haben die Lerner die Wörter über die Tafeln ständig präsent, und ein Abruf der Vokabeln kann durch einen Blick auf die Tafeln erfolgen. Im fortgeschritteneren Unterricht werden mit Hilfe von Bildern und Arbeitsblättern semantische Beziehungen zwischen lexikalischen Einheiten erarbeitet, deren Erfassen zusammen mit der Kenntnis grammatischer Regeln die Produktion von Aufsätzen und die Lektüre literarischer Texte ermöglichen soll (GATTEGNO 1978). Silent Way bringt zweifelsohne viel Abwechslung in den Fremdsprachenunterricht. Das Lehrkonzept sollte aber wegen der Komplexität der sprachlichen Lernprozesse, die es nur zum Teil berücksichtigt, eher als eine Ergänzung anderer Lehrmethoden betrachtet werden.

\section{Total Physical Response}

Total Physical Response (,Vollständige körperliche Reaktion') wurde von JAMES J. ASHER (1966) entwickelt. Nach Asher kann ein effizientes Lernen erst dann stattfinden, wenn der Schüler die zielsprachlichen Strukturen so aufnimmt, wie das Kind seine Muttersprache erwirbt. Dies geschieht wiederum dadurch, dass die Kinder die Äußerungen der Erwachsenen hören, die vor allem Befehle, Anordnungen sowie Anweisungen beinhalten, um dann entsprechende Handlungen ausführen. Durch die bei der Ausführung der Befehle vorkommenden Fehler eignen sie sich überdies die situationellen Bedingungen für die korrekte Verwendung solcher Äußerungen an. Analog dazu werden auch den Lernern von der Lehrperson Befehle erteilt und gleichzeitig ausgeführt. 
Die Befehle können z.B. lauten Stehen Sie auf, Setzen Sie sich, Machen Sie das Buch auf usw. Zuerst schauen die Schüler nur zu. Nach drei bis vier Demonstrationen führen sie den Befehl gemeinsam mit der Lehrperson aus, dann auch ohne den Lehrer. Nach weiteren Wiederholungen der Befehle sind die Lerner schließlich dazu befähigt, die Rolle der Lehrperson zu übernehmen und selbst Befehle zu erteilen. Der Unterricht wird in Aufforderungen fortgeführt, auch nachdem die Schüler ohne Aufforderung seitens des Lehrers zu sprechen anfangen (ASHER 1966; ASHER 1982; WIENOLD 1991). Asher legt dar, dass eine wichtige Voraussetzung für den gelungenen Verlauf des Lernprozesses eine entspannte Atmosphäre darstellt, in der die Schüler ihre Emotionen freisetzen und spontan sprachlich reagieren können. Unter diesen Umständen greift der Lerner auf die biologischen Spracherwerbsmuster zurück, die nach ASHER (1982:2-18) beim Erwachsenwerden nicht verschwinden. Die Spontaneität des fremdsprachlichen Verhaltens ermöglicht eine vertiefte multimodale Analyse des Sprachmaterials nicht nur in der linken, sondern in beiden Gehirnhälften. Der Unterricht kann verschiedenartig variiert werden, u. a. durch abwechselnd leises und lautes Sprechen durch den Lehrer, durch Angabe von Übersetzungen, durch Aufforderung zum lauten Nachsprechen im Chor. Der Lernprozess wird dabei durch den so genannten Mönchsgang stimuliert: Lehrer und Lernende schreiten in einem Kreis und führen bestimmte Gesten aus, mit denen die zu semantisierenden lexikalischen Strukturen nachgeahmt werden sollen (SCHIFFLER 2002:44).

Das Lehrkonzept sieht außer dem beschriebenen Lehrverfahren keine besonderen Formen der Wortschatzarbeit vor. Die Vermittlung des zielsprachlichen Wortschatzes beschränkt sich folglich nur auf relativ unkomplizierte lexikalische Strukturen. Die Nachahmung des natürlichen L1-Erwerbs durch die Verbindung der Lernprozesse mit dem Ausführen von Bewegungen lässt auch außer Acht, dass der kindliche Spracherwerb eine äußerst komplexe kognitive Struktur aufweist und mit einem individuell geprägten Sozialisierungsprozess einhergeht. Vor diesem Hintergrund gesehen muss die Bedeutung der Befehle für die sprachliche Entwicklung des Kindes relativiert werden: Sie sind nur einer unter vielen anderen Stimuli und Erfahrungen, die die sprachliche, emotionale und soziale Entfaltung des Kindes beeinflussen (MEIBAUER / DEMSKE / GEILFUB-WOLFGANG / PAFEL / RAMERS / ROTHWEILER / STEINBACH 2002:264270, 284-290). Es kann somit nicht verwundern, dass empirische Untersuchungen zur lexikalischen Effizienz der Total Psysical Response-Methode keine Überlegenheitseffekte dieses Lehrkonzeptes nachweisen konnten (STORK 2003:124f., 172f.). 
Wortschatzarbeit in alternativen Lehrmethoden - eine kritische Betrachtung

\section{Community Language Learning}

Im Community Language Laerning (,Gemeinschaftliches Sprachenlernen“) des Psychotherapeuten CHARLES A. CURRAN (1972) werden besonders stark die Entfaltung und der Erhalt der emotionalen Seite des Lerners gefördert. Der Lerner wird als ein Individuum betrachtet, das eine Fremdsprache zu beherrschen vorhat, die eine ebensolche Singularität besitzt. Die Berücksichtigung der Emotionen sowie der persönlichen Lernereinstellungen und -eigenschaften soll die Freisetzung erheblicher kognitiver Kräfte zur Folge haben. Damit aber der Lernprozess wirklich in einer stressfreien Atmosphäre verlaufen kann, verlangt Curran vom Lehrer sehr viel Bereitschaft, sich zurückzunehmen, bis hin zur Passivität. „Es ist ein Lehrer, der da ist, wenn er gebraucht wird, aber sonst wenig sagt. Er ist möglichst überhaupt nicht Autorität.“ (WIENOLD 1991:354) Er tritt als Sprachberater (,language counselor") jeweils hinter den Lerner (,client'), der sich äußern will, und übersetzt leise seine muttersprachlichen Äußerungen in die Fremdsprache. Der Schüler wiederholt die Übersetzungen. Erst allmählich beginnt der ,client' selbständig Aussagen in der Fremdsprache zu produzieren, wobei sich der Lehrer mit Korrekturen zurückhält, bis der Lerner größeres Selbstvertrauen gewinnt, das es ihm erlaubt, Verbesserungen hinzunehmen. Die Verbesserungen zielen folglich nicht nur darauf ab, Fehler zu korrigieren, sondern tragen auch zur Erweiterung des Wortschatzes bei, indem Idiome und elegantere Ausdrücke hinzugefügt werden (SCHWERDTFEGER 1983:3f.). Die fremdsprachlichen lexikalischen Strukturen werden nicht zuletzt im Rahmen von Gruppenarbeit analysiert und beherrscht. Das zu analysierende Material besteht dabei aus Tonbandaufzeichnungen der Lerneräußerungen. Die Resultate der Gruppenarbeit werden im Forum unter Teilnahme des Lehrers ausgewertet. Sowohl während der Gruppenarbeit als auch im Forum kann neben der Fremdsprache die Muttersprache verwendet werden. Community Language Learning ermöglicht einen stufenweisen Einstieg in die zielsprachlichen - darunter lexikalischen - Strukturen. Das Lehrund Lernverfahren umfasst insgesamt fünf Stadien, die mit einer Phase der so genannten maximalen Sicherheit des Lerners anfangen und zum freien Gebrauch der Zielsprache führen. Eine nicht unbedeutende Rolle kommt im Lernprozess der Muttersprache und interlingualen Vergleichen zu. Die letztgenannten werden vor allem bei der kontrastiven Analyse von Übersetzungen zu Hilfe gezogen (CURRAN 1972). Trotz vieler Vorteile dieser alternativen Methode stellt die zu starke Berücksichtigung der emotionalen Seite des Lerners eine gewisse Unzulänglichkeit dar, weil sie in den frühen Unterrichtsphasen 
Korrekturen durch den Lehrenden praktisch ausschließt, was nicht selten falsche Semantisierungsprozesse zur Folge haben kann.

\section{Abschließende Bemerkungen}

Aus den obigen Ausführungen wird ersichtlich, dass die alternativen Lehrmethoden kein Allheilmittel gegen Lernprobleme jeder Art - also auch gegen solche in der Wortschatzarbeit - darstellen und folglich den in der Unterrichtspraxis etablierten Lehrkonzepten keinesfalls überlegen sind. An dieser Feststellung würde auch die Analyse anderer, hier außer Acht gelassener moderner Lehrverfahren, wie z. B. Confluent Approach (,Sprache von innen') von BEVERLY GALYEAN (1979) oder Delay in Oral Practice (,Verzögertes mündliches Üben') von VALERIAN O. Postovsky (1981), kaum etwas ändern. Das unbestreitbare Verdienst der alternativen Lehrmethoden liegt ganz sicher im Hervorheben der emotiven Seite des fremdsprachlichen Lernprozesses. Die eigenen Bedürfnisse, Interessen, Werte und augenblicklichen Handlungen der Lernenden treten in den Vordergrund. Die Lerner werden oft sogar dazu angehalten, über ihre emotionalen Zustände zu sprechen. Weil auf diese Weise das Individuum des Lernenden ins Zentrum des Lerngeschehens rückt, werden die alternativen Lehrkonzepte oft auch als humanistische Ansätze bezeichnet. Die neuesten neuro- und psycholinguistischen Forschungen bestätigen im Übrigen eindeutig die Wichtigkeit der Freisetzung von Emotionen für den erfolgreichen Verlauf der Lernprozesse und für die dauerhafte mentale Verankerung neuer Erfahrungen, wobei angemerkt werden muss, dass sich Kognition und Emotion gegenseitig förderlich beeinflussen (HÜTHER 2004). Auch der Fremdsprachenunterricht scheint gerade dann zu den besten Resultaten zu führen, wenn ein Zusammenspiel der kognitiven und emotiven Faktoren stattfindet (WOLFF 2004) und positive Attitüden zu der zu erlernenden Fremdsprache hergestellt werden (LOCHTMAN / LUTJEHARMS 2004).

Eine gewisse Schwäche der modernen Methoden ist folglich nicht darin zu suchen, dass sie in unzureichendem Maße die emotionelle Individualität des Lerners berücksichtigen, sondern eher in einer allzu eingeschränkten Betrachtungsperspektive der fremdsprachlichen Lernprozesse. Die Autoren der Lehrkonzepte übersehen die unüberschaubare Komplexität der kognitiven Seite des gesteuerten Sprachenerwerbs. Diese vielschichtige Strukturiertheit der fremdsprachlichen Lernprozesse tritt besonders deutlich bei der Wortschatzarbeit hervor: Der Lerner baut das fremdsprachliche mentale Lexikon auf, indem er nach den muttersprachlichen lexikalischen Entitäten greift, mentale Wörternetze aufbaut (Wortfamilien, Synonyme, Antonyme, Kohypo- 
Wortschatzarbeit in alternativen Lehrmethoden - eine kritische Betrachtung

nyme, Sachfelder, mentale lexikalische Scripts usw.), sich während der Arbeit mit authentischen Texten Kollokationen und manchmal sogar ganze Sätze merkt sowie beim Konstruieren eigener geschriebener oder gesprochener Texte schöpferisch mit seinem fremdsprachlichen Vokabular umgeht. Oft nutzt der Lernende auch die Kenntnisse weiterer ihm bekannter Sprachen. Der Lernvorgang weist außer seiner komplexen Struktur eine große Individualität auf, die von einem Lerner zum anderen stark variieren kann. Vor diesem Hintergrund scheint am effizientesten eher ein Fremdsprachenunterricht zu sein, in dem neben der Freisetzung der Emotionen auch individuelle Lernstile, die förderliche Rolle der Muttersprache, natürliche Erwerbssequenzen sowie der komplexe Charakter der kognitiven Erwerbsprozesse berücksichtigt werden (BUTZKAMM 2002:170-196; NEVELING 2004). Eine wichtige Bedeutung kommt in diesem Zusammenhang der Lernerautonomie und dem interkulturellen Lernen zu, die den Schüler dazu befähigen sollen, selbständige Lernstrategien zu entwickeln und die Fremdsprache auch als ein spezifisches gemeinschaftliches Kulturgut $\mathrm{zu}$ sehen (LUCHTENBERG 2001). In einem so betrachteten Fremdsprachenunterricht können die alternativen Methoden selbstverständlich ab und zu als Teil des multimodalen Lernprozesses eingesetzt werden und als eine Art Abwechslung im schulischen Unterrichtsalltag zu Lernerfolgen führen.

\section{Literatur}

ASHER, JAMES J. (1966): The Learning Strategy of Total Physical Response. A Review. In: Modern Language Journal 50:79-84.

- (1977 / 21982): Learning another language through actions: the complete teacher's guidebook. Los Gatos.

BAUR, RuPPRECHT S. (1990 / $\left.{ }^{2} 1991\right)$ : Superlearning und Suggestopädie. Grundlagen, Anwendung, Kritik, Perspektiven. Berlin.

- (1991a): Die Suggestopädie - eine neue Methode der Fremdsprachenvermittlung. In: BATZ, Richard / BufE, WolfGAng (eds.): Moderne Sprachlehrmethoden. Theorie und Praxis. Darmstadt, 291-315.

BOHN, REINER / SCHREITER, INA (2000): Wortschatzarbeit in den Sprachlehrwerken Deutsch als Fremdsprache: Bestandsaufnahme, Kritik, Perspektiven. In: KÜHN, PETER (ed.): Studien zu Deutsch als Fremdsprache V. Wortschatzarbeit in der Diskussion. Hildesheim/Zürich/New York, 57-98.

BÖRner, WolfGAng / Vogel, Klaus (eds.) (2004): Emotion und Kognition im Fremdsprachenunterricht. Tübingen.

BRÖHM-OFFERMANN, BIRGIT (1989 / $\left.{ }^{3} 1994\right)$ : Suggestopädie - sanftes Lernen in der Schule. Lichtenau. 
Lesław Tobiasz

ButZKamm, Wolfgang (1989/32002): Psycholinguistik des Fremdsprachenunterrichts. Tübingen/Basel.

Curran, Charles A. (1972): Counseling-learning. A whole-person model for education. New York.

Galyean, Beverly (1979): A confluent approach to curriculum design. In: Foreign Language Annals 12:121-128.

Gattegno, CALEB (1978): Teaching foreign languages in schools. The silent way. Reading.

HOLTWISCH, HERBERT (1994): Behalten suggestopädisch unterrichtete Schulkinder ihren Lernstoff besser? In: Neusprachliche Mitteilungen aus Wissenschaft und Praxis 47/4:228-233.

HÜTHER, GERALD (2004): Die Bedeutung emotionaler Reaktionen für Lernprozesse und die Verankerung neuer Erfahrungen. In: BÖRNER / VOGEL, 25-34.

Lewicki, Roman E. (1990): Innowacyjność w sugestopedycznym nauczaniu języków obcych. [Innovation im suggestopädischen Fremdsprachenunterricht]. Wrocław.

Lochtman, Katja / LutJEhaRms, MAdELINE (2004): Attitüden zu Fremdsprachen und zum Fremdsprachenlernen. In: BÖRNER / VOGEL, 173-189.

LuCHTENBERG, Sigrid (2001): Language(s) and Cultural Awareness: Ein Thema für die Fremdsprachenlehrerausbildung? In: Neusprachliche Mitteilungen 54/3:130-138.

MeIbAuer, Jörg / Demske, Ulrike / GeIlfuss-WolfGANG, Jochen / PAFEl, JÜrgen / Ramers, Karl Heinz / Rothweiler, Monika / Steinbach, Markus (2002): Einführung in die germanistische Linguistik. Stuttgart/Weimar.

NeVEling, Christiane (2004): Wörterlernen mit Wörternetzen. Eine Untersuchung zu Wörternetzen als Lernstrategie und als Forschungsverfahren. Tübingen.

POSTOVSKY, VALERIAN O. (1981): The priority of aural comprehension in the language acquisition process. In: WINITZ, HARRIS (ed.): The comprehension approach to foreign language instruction. Rowley (Mass.), 170-187.

Riedel, Katja (2000): Persönlichkeitsentfaltung durch Suggestopädie. Suggestopädie im Kontext von Erziehungswissenschaft, Gehirnforschung und Praxis. Baltmannsweiler.

SCHIFFLER, LUdGER (1989): Suggestopädie und Superlearning - empirisch geprüft. Frankfurt (M.).

- (2002): Fremdsprachen effektiver lernen und lehren. Beide Gehirnhälften aktivieren. Donauwörth.

SCHWERDTFEGER, INGE CH. (1983): Alternative Methoden der Fremdsprachenvermittlung für Erwachsene. Eine Herausforderung für die Schule? In: Neusprachliche Mitteilungen aus Wissenschaft und Praxis 36:3-14.

STORK, ANTJE (2003): Vokabellernen. Eine Untersuchung zur Effizienz von Vokabellernstrategien. Tübingen. 
Wortschatzarbeit in alternativen Lehrmethoden - eine kritische Betrachtung

WIENOLD, GÖTZ (1991): Zu einigen innovativen methodischen Konzepten im Fremdsprachenunterricht: Total Physical Response, Silent Way und Community Language Learning. In: BATZ, RiCHARD / BUFE, WOLFGANG (eds.): Moderne Sprachlehrmethoden. Theorie und Praxis. Darmstadt, 326-371.

WOLFF, DIETER (2004): Kognition und Emotion im Femdsprachenerwerb. In: BÖRNER / VOGEL, 87-98. 